\title{
Ist die Bezeichnung "Verhaltenstherapie» noch zeitgemäß?
}

\author{
Michael Linden \\ Rehabilitationszentrum Seehof der Deutschen Rentenversicherung Bund, Teltow/Berlin, Deutschland
}

Folgt man dem aktuellen fachpolitischen Diskurs, so scheinen Begriffe wie «integrative Psychotherapie», «allgemeine Psychotherapie», «störungsspezifische Psychotherapie», «psychologische Psychotherapie», «ärztliche Psychotherapie», «mehrdimensionale Psychotherapie», «ganzheitliche Psychotherapie» usw. immer mehr Zustimmung zu finden. Wer sich für eine «schulenspezifische» Psychotherapie einsetzt, gerät in Argumentationsnot. Es stellt sich damit auch die Frage, ob die Bezeichnung «Verhaltenstherapie» nicht allmählich anachronistisch wird und man an Ansehen gewinnen könnte, wenn man stattdessen von «evidenzbasierter Psychotherapie» oder Ähnlichem spräche.

Ich bekenne, dass ich für die Beibehaltung der Bezeichnung «Verhaltenstherapie» bin. Hierfür gibt es eine Reihe von Gründen, die ich im Folgenden kurz nennen will, ohne sie im Detail ausführen zu können.

Zunächst einmal ist an wissenschaftstheoretische Selbstverständlichkeiten zu erinnern: Die Begriffe, mit denen in der Psychotherapie operiert wird, sind hypothetische Konstrukte, die keine «Dinge» kennzeichnen, sondern nur im Rahmen der zugrunde liegenden Theorien verstehbar sind. Es ist Ausdruck undisziplinierten Denkens, wenn Verhaltenstherapeuten z.B. mit dem Begriff «Widerstand» operieren, der nur auf dem Hintergrund der Theorie der Übertragung verstehbar ist. Verhaltenstherapeuten sollten stattdessen fragen, welche Kontingenzen bedingen, dass der Patient nicht tut, was der Therapeut will. Ebenso wenig ist es akzeptabel, wenn Tiefenpsychologen «Expositionen» durchführen, ohne dies in die Lerntheorien einzubetten. Nicht selten besteht das Ergebnis eines nicht theoriegeleiteten Vorgehens schlicht darin, $\mathrm{Pa}$ tienten zu erschrecken. Das Herausnehmen theoretischer Konstrukte aus ihrem Theoriekontext ist in der Wissenschaftstheorie als «Reifizierung» beschrieben und gilt als Denkfehler. Dies entspricht auch der Beobachtung in der Praxis: Je unbekümmerter mit theoretischen Begriffen hantiert wird, desto weniger scheinen die Betreffenden tatsächlich die dahinter liegenden Definitionen zu kennen.

Genauigkeit bei der Benutzung von theoretischen Konstrukten und Behandlungstheorien ist auch unverzichtbar, wenn es um die praktische Therapie geht. Die in den Psychotherapieschulen ausgearbeiteten Theorien menschlichen Verhaltens stellen heuristische Modelle dar, die es erlauben, Krankheiten zu beschreiben, und deren zugrunde liegende Annahmen und empirische Untersuchungen Handlungsansätze aufzeigen, um Krankheiten zu bessern. Ein wesentlicher Wirkmechanismus von Psychotherapie ist, dass sie den Patienten Funktionsmodelle ihrer Störungen vermittelt, aus denen sie dann Modifikationsansätze ableiten können. Wenn dies gelingen soll, ist auch hier terminologische und theoretische Klarheit erforderlich. Es nicht zielführend, solche Erklärungsmodelle dadurch zusätzlich komplex zu machen, dass viele Theorien neu zusammengemischt werden. Sprachliche, konzeptuelle und theoretische Eindeutigkeit sind erst recht dann erforderlich, wenn mehrere Therapeuten mit einem Patienten zusammenarbeiten, wie es z.B. in Kliniken geschieht. Es ist nicht nachvollziehbar, wie sich Kliniken gelegentlich damit brüsten, dass ihre Therapeuten in unterschiedliche Richtungen arbeiten. Die praktische Erfahrung lässt für den Patienten bei dieser Art von Theorie-Potpourri nichts Gutes erwarten.

Dies leitet zum Problem der Qualitätssicherung über. Die Einleitung einer Therapie und letztlich auch ihre Bezahlung durch die Kostenträger ist nur gerechtfertigt, wenn es guten Grund zu der Annahme gibt, dass es dem Patienten am Ende der Behandlung besser gehen wird als vorher. Diese ethische wie ökonomische Voraussetzung einer Behandlung wird in der Regel dadurch geschaffen, dass ein Therapeut sagt, er wende bestimmte erprobte Therapiemethoden an, und die in diesem Kontext erarbeiteten Evidenzen für sich selbst reklamiert. Dies bedeutet aber auch, dass der Therapeut verpflichtet ist, sich an die von ihm als Referenz herangezogenen Vor-

\begin{tabular}{ll}
\hline KARGER & @ 2007 S. Karger GmbH, Freiburg \\
Fax +497614520714 & Accessible online at: \\
$\begin{array}{l}\text { E-mail Information@Karger.de } \\
\text { www.karger.com }\end{array}$ & www.karger.com/ver
\end{tabular}

Prof. Dr. Michael Linden

Rehabilitationszentrum Seehof der Dt. Rentenversicherung Bund Lichterfelder Allee 55

14513 Teltow/Berlin, Deutschland

Tel. +49 3328 345678, Fax 345555

E-mail michael.linden@zedat.fu-berlin.de 
gehensweisen zu halten. Man kann nicht Wein verkaufen und Zuckerwasser liefern. Insofern tun Krankenkassen, Unfalloder Rentenversicherungsträger gut daran, zumindest eine Schulenspezifität von den Therapeuten zu verlangen und es nicht in das Belieben des Einzelnen zu stellen, seine persönlichen Einfälle als Therapie zu verkaufen.

Die Frage ist, ob die Qualitätssicherung nicht über Konzepte einer «störungsspezifischen» Psychotherapie noch besser zu leisten wäre als über die Vorgaben einer «schulenspezifischen» Therapie. Dem steht die Vielgestaltigkeit der Realität entgegen. Letztlich werden in der Psychotherapie nicht Krankheiten, sondern Kranke behandelt. Dabei gilt, dass auch die Durchführung einer störungsspezifischen Psychotherapie eine verfahrensspezifische Grundausbildung benötigt. So wie ein Chirurg je nach Schule entweder Schneiden und Knoten oder aber ein Endoskop steuern können muss, so muss ein Psychotherapeut über «therapeutische Basisfertigkeiten» verfügen, die konstituierend für unterschiedliche Therapieschulen sind. Therapeutische Schulen lassen sich über verschiedene Sets an Basistechniken definieren, die jeder Therapeut der entsprechenden Schule beherrschen muss und die störungsübergreifend zur Anwendung kommen. In der Verhaltenstherapie sind dies z.B. das Hausaufgabenstellen oder die Analyse automatischer Gedanken, in der psychodynamischen Psychotherapie sind es die Übertragungsanalyse oder die Deutung.

Das Erlernen der schulenspezifischen Basisfertigkeiten ist auch der Kern jeder Aus- und Weiterbildung zum Psychotherapeuten. Im Verlauf seiner Aus- und Weiterbildung zum Psychotherapeuten behandelt ein Arzt oder Psychologischer Psychotherapeut etwa ein Dutzend Patienten, er lernt also nur einen sehr geringen Teil der Störungen kennen, die er in seiner weiteren beruflichen Laufbahn behandeln wird. Eine Ausbildung in störungsspezifischen Psychotherapien ist faktisch nicht machbar. Dennoch wird jeder Therapeut später unterschiedlichste Krankheiten «störungsspezifisch», auf dem Boden «seiner» Therapieschule behandeln. Wollte man in der Ausbildung eine Tour d'Horizon über alle Therapieschulen oder störungsspezifischen Ansätze machen, so käme das dem Versuch gleich, einen Musiker dadurch auszubilden, dass man ihm fünf Stunden Klavierunterricht, fünf Stunden Querflötenunterricht usw. erteilt, statt die zur Verfügung stehenden 100 Unterrichtsstunden auf die Blockflöte zu verwenden.

Selbst wenn man einem störungsspezifischen Manual wie z.B. der Schematherapie folgt, muss dennoch eine schulenspezifische Basiskompetenz vorliegen. Nur wer weiß, was mit automatischen Gedanken und Schemata im kognitiven Modell gemeint ist und dies in der Operationalisierung nicht mit «Gedanken» verwechselt, kann die Schematherapie auch störungsspezifisch korrekt umsetzen. Hier geht es um die Qualität des einzelnen Therapeuten. Selbst wenn ein Therapeut vorgibt, Verhaltenstherapie oder Psychoanalyse durchzuführen, bleibt die Frage, ob er es auch kann. Es gilt die Tennisspielerregel: «Gut gewollt ist nicht gleich gut gemacht.» Will man feststellen, ob ein Therapeut gut oder schlecht ist, bedarf es ebenfalls einer schulenbezogenen Präzisierung. Es ist am Einzelfall zu prüfen, ob ein Psychotherapeut, der vorgibt, Verhaltenstherapie zu machen, z.B. überhaupt Hausaufgaben einsetzt (wenn nicht, ist er kein Verhaltenstherapeut) und ob er es richtig macht (wenn nicht, ist er kein guter Verhaltenstherapeut). Empirische Untersuchungen zeigen, dass eine hohe Therapeutengüte nicht selbstverständlich ist und auch nicht mit langer Berufserfahrungen von alleine kommt. Hier gilt nämlich die Golferregel: 'Not practice makes perfect, perfect practice makes perfect!'

Zusammenfassend sind Therapeuten, die einer ganzheitlichen oder integrativen Psychotherapie das Wort reden, zunächst einmal nachweispflichtig, was die von ihnen benutzten Begriffe eigentlich bedeuten sollen, was sie in der Therapie überhaupt machen und womit sie die Behauptung begründen, dass das gut sein soll. In diesem Heft finden Sie ein Interview mit Prof. Aaron T. Beck, dem Doyen der Kognitiven Therapie. Seine Antworten machen deutlich, dass er von einem Schulenpotpourri wenig hält. Er votiert für Klarheit in den theoretischen Modellen als wichtige Voraussetzung für eine transparente qualitativ hochwertige und evidenzbasierte Psychotherapie. Wenn die «Verhaltenstherapie» ihren Namen ändern sollte, dann wäre über den präzisierenden Zusatz «kognitiv» nachzudenken. 